\title{
The cystic fibrosis transmembrane recruiter the alter ego of CFTR as a multi-kinase anchor
}

\author{
Anil Mehta
}

Received: 9 August 2006 / Accepted: 11 May 2007 / Published online: 6 September 2007

(C) Springer-Verlag 2007

\begin{abstract}
This review focuses on a newly discovered interaction between protein kinases involved in cellular energetics, a process that may be disturbed in cystic fibrosis for unknown reasons. I propose a new model where kinasemediated cellular transmission of energy provides mechanistic insight to a latent role of the cystic fibrosis transmembrane conductance regulator (CFTR). I suggest that CFTR acts as a multi-kinase recruiter to the apical epithelial membrane. My group finds that, in the cytosol, two protein kinases involved in cell energy homeostasis, nucleoside diphosphate kinase (NDPK) and AMP-activated kinase (AMPK), bind one another. Preliminary data suggest that both can also bind CFTR (function unclear). The disrupted role of this CFTRkinase complex as 'membrane transmitter to the cell' is proposed as an alternative paradigm to the conventional ion transport mediated and CFTR/chloride-centric view of cystic fibrosis pathogenesis. Chloride remains important, but instead, chloride-induced control of the phosphohistidine content of one kinase component (NDPK, via a multi-kinase complex that also includes a third kinase, CK2; formerly casein kinase 2). I suggest that this complex provides the necessary near-equilibrium conditions needed for efficient transmission of phosphate energy to proteins controlling cellular energetics. Crucially, a new role for CFTR as a kinase controller is proposed with ionic concentration acting as a signal. The model posits a regulatory control relay for energy sensing involving a cascade of protein kinases bound to CFTR.
\end{abstract}

A. Mehta $(\varangle)$

Department of Maternal and Child Health Sciences,

University of Dundee, Ninewells Hospital,

Dundee DD1 9SY, UK

e-mail: a.mehta@dundee.ac.uk
Keywords NDPK · Drosophila AWD ·

Energy metabolism $\cdot$ Kinase $\cdot$ AMPK $\cdot$ CK2

\section{Introduction}

James Watt had a problem. His steam engines kept slowing down on hills due to a mismatch between energy transmission and the extra gravity-induced load from the hill. He solved this by creating a feedback loop between the rate of engine turnover and the steam valve (http://oldenginehouse. users.btopenworld.com/technical.htm), thus creating a 'Watt governor' to match energy input to the applied load. This review describes progress towards understanding a cellular equivalent, balancing supply and demand of cell energy.

The focus is on epithelial (dys)function in cystic fibrosis (CF) as a paradigm for governor dysfunction. The governor concept necessitates new networking roles for novel protein kinase complexes as molecular transmission grids comprising heterodimers of 'well understood' single protein kinases [nucleoside diphosphate kinase (NDPK), AMP-activated kinase (AMPK) and CK2 (formerly casein kinase 2)] such that their combined activities as 'Watt governor complexes' differ from the sum of their parts. The focus then moves to the cell membrane to demonstrate how the cystic fibrosis transmembrane conductance regulator (CFTR) fits into the picture via its membrane-delimited interaction with the third and constitutively active kinase, CK2. The review concludes with the proposal that CFTR acts as a transmembrane recruiter of multiply interacting protein (kinase) transducers of cell energy to the apical membrane of epithelial cells.

It is not the purpose of this review to cover all the proteins reported to bind to CFTR, as these areas have been 
described by others very recently [21]. Neither is one related aspect discussed in this paper, namely, the exact role of chloride concentration in the histidine phosphorylationdependent processes described below. As discussed by us elsewhere [49], chloride itself can play a signalling role recently because its intracellular concentration (typically $40 \mathrm{mM}$ ) is often counter-balanced against the prevailing resting trans-membrane voltage such that a change in that voltage will either promote chloride accumulation or result in intracellular chloride depletion depending on the direction of voltage change (depolarisation or hyperpolarisation).

Energy governors and cell function The key role of cellular energy in disease pathogenesis and cell death has been emphasised recently in relation to Huntingdon's disease [45]. Shapiro [46] long ago suggested similar deficiencies in cystic fibrosis. Epithelial cells expend a significant proportion of the $38 \mathrm{~kJ} / \mathrm{mol}$ of adenosine triphosphate (ATP)-derived energy liberated per hydrolytic cycle of gamma phosphate cleavage to maintain ion gradients across the outward-facing apical plasma membrane and the internal-facing basolateral membrane. This 'gradientreserve' of ionic energy is akin to pumping water to the top of a mountain reservoir ready to drive turbines when electricity is required at high demand. This gradient reserve must additionally be flexible enough to drive cellular transport functions at rest, after cell stimulation, during cell stress and after recovery. For ill-understood reasons, many of these processes are disordered in the common inherited disease CF where the disease-causing protein (CFTR) is mutated [21, 46]. Confusingly, proteins such as the epithelial sodium channel $(\mathrm{ENaC})$ are hyperactive, and the multiplicity of defects in unrelated proteins induced by CFTR mutation has been recently reviewed elsewhere by us $[30,31,49]$ and others [21]. To govern the gradient reserve in epithelial cells, there is evidence that the apical and basolateral membrane protein channels receive cytoplasmic feedback signals $[15,20]$. It is clear for example that such a feedback loop controls trans-apical, G-protein regulated, CFTR-dependent, ion transport as described by Schwiebert et al. [43] and further investigated in terms of ion concentration as the signal to (an uncharacterised) G-protein function by Komwatana et al. [26, 13]. These data have never been followed up in detail, but the recent work of Wieland [52] generates new insight by revealing how $G$ proteins, their histidine phosphorylation (via NDPK) and enhanced guanosine triphosphate (GTP) flux to the membrane might be linked to one another. These data suggest basal mechanisms to synthesise cyclic AMP (cAMP) constitutively [52]. That data combined with our own and that of others suggest that ionic concentration inside the cell (sodium or chloride) negatively governs apical ion entry, but the mechanism is a complex mix of ion-dependent and independent mechanisms [40]. Although the underlying ionic feedback principle is similar at the basolateral membrane, the controlling proteins differ. For example, Gimenez and Forbush [20] find that the cytosolic chloride concentration provides a feedback signal to restrain sodiumdriven, inwardly directed 'accumulative' chloride co-transport through the basolateral membrane via a chloride-sensing protein kinase that inhibits the $\mathrm{Na} / 2 \mathrm{Cl} / \mathrm{K}$ cotransporter [15]. The details are described elsewhere [20], but the combination of observations is consistent with the presence of the components of a Watt governor in epithelial cells-where each component may be a kinase or a regulator of a kinase (which may itself be a scaffold protein, a kinase or phosphatase).

Returning to the mountain and water pump analogy, what happens if the pump keeps driving water up hill when energy demand requires downward flow to generate electricity? The effects of such futile cycling are seen when CFTR is mutated $[34,46,48]$ with the result that energy expenditure is inappropriately elevated at rest. Some of the mechanisms are reviewed by Milla et al. [32] and Moran et al. 33] and by my own group [47], which follow on from the seminal studies of Shapiro [46] almost two decades ago. Our recent work and that of Moran's group [32] shows that, in $\mathrm{CF}$ females, diabetes and respiratory epithelial dysfunction combine to create early mortality relative to males. It is as if the extra energetic demand of CF-related diabetes leads to catastrophic failure of a (already deranged) female pulmonary epithelial 'engine'. It is also important not to forget the extra load imposed by CF epithelia compensating for excessive sodium absorption that accompanies mutant CFTR (see [26, 30] for example and the papers from Puntheeranurak et al. [38]), but the role of female sex and or hormones, which can have significant effects on ion transport [38], remains to be determined. Conversely, malespecific hormones may be protective in this respect.

Molecular insight into cellular transmission grids How does a cell transmit energy to its working parts - the proteins in a cytosolic soup with $300 \mathrm{mg} / \mathrm{ml}$ of mixed proteins? Specifically, how is it possible to promote the signal fidelity of energy transmission as gamma phosphate of ATP given the diffusion limits and high inter-molecular collision rates imposed by the crowded cytosol? To avoid energy dissipation and consequent electrical blackout, cellular energy supply must be transmitted (channelled) to control the activity of proteins mediating ion influx and exit but without signal attenuation. This is not a trivial problem, as those at the cutting edge of understanding ionic conductivity in densely packed environments [51] do not (to my knowledge) frequently interact with cell biologists or epithelial ion transport specialists. In equal measure, the relationship between that process and ion transport at a 
single channel level suffers from problems in the interpretation of the underlying theory [25]. Progress is being made [44], but Dzeja and Terzic [17] have circumvented these interpretative difficulties by adopting a gene knockout approach. They invoke the analogy of the electricity grid, where the power station (mitochondrion) links via pylons (networks of enzymes near equilibrium that form fixed subcellular arrays acting as molecular relays-NDPK is one component) to send energy waves (protons and/or phosphate) through the cytoplasm without signal dissipation (energy loss as random motion and heat imposed by cytosolic diffusion limits). A good analogy for the nearequilibrium requirement is that of an elevator, which minimises power usage by driving a counterweight during its operation, thus only expending marginal energy proportional to the weights of the elevator occupants per lift cycle. They suggest that by utilising intracellular protein tunnels, insulated from the molecular chaos in the cytosolic 'earth', these protein-protein networks manifest novel properties that differ from their individual roles. For example, a vectorial ion smuggling model is proposed for carbonic anhydrase (CA) as a relay of multimers that dispose of locally generated protons. These authors propose that accumulation of protons during energy generation would otherwise create a build up of voltage-counteracting energy flow at the site of generation by inhibiting actomyosin ATPase activity for example. This tunnelling phenomenon is a form of 'substrate channelling' whereby the substrate (proton here, phosphate in other cases) never 'sees' the cytosol (these are the transmitters of force, the equivalent of shafts, cams, gears and pulleys of the Watt governor). However, such circuits can be set up in linear or parallel arrays (multiple governors). Dzeja et al. [18] report that knock out approaches show how these networks act like a true parallel transmission grid with latent compensatory increments in activity of other grid members when one member is knocked out. This grid idea is supported by work undertaken in other laboratories [24]. For example, it may be that the energy transmitted by mitochondrial ATP synthesis to the nucleus is essential for all nuclear transport to function. Such transport requires mitochondrial energy supply to be coupled to both GTP and ATP synthesis at the nuclear membrane (glycolysis alone is insufficient). Whilst the proximity of mitochondria to the outer nuclear membrane helps to reduce diffusion-induced dissipation, it is an adenylate kinase relay (AKR) that normally maintains energy density as ATP and AMP and ADP ratios. Upon AKR knockdown, a parallel creatine kinase relay (CKR) substitutes, and nuclear transport resumes at baseline. However, after further cell stress, this compensatory relay fails [18]. This leads to the notion that a cell with one defective relay may look superficially normal at baseline but only reveals its compensatory state by failing under cellular stress - a paradigm for CF? My personal observations of the post-infection, rapid weight decline in $\mathrm{CF}$ patients together with their epidemiological data [47], suggest that $\mathrm{CF}$ cells share this property of superficial normality coupled to stress-induced failure because one or more latent CFTR-dependent functions are defective when CFTR is mutated. One possibility is that females with CF succumb to the additional stress of CF-related diabetes by this route (see above) because of the extra demands on female fat metabolism after puberty. NDPK is known to control ATP citrate lyase, a key controller of fat metabolism for example [7-9]. The ability to withstand cell stress after infection for example is the province of an energy sensor, activated by AMP, AMP-activated kinase (AMPK) [23, 28], but we find that this kinase binds NDPK (function unknown) [37]. Note that AMP-dependent kinase is a misnomer and should not be used due to the substantial basal activity of AMPK, and with the above network role in mind, that adenylate kinase is needed for AMPK to work efficiently [23]. Further discussion may be found elsewhere $[17,18,34]$.

Cell energy balance after power failure The macroscopic AKR/CKR grid described above eventually meets the protein equivalent of a substation and power-down transformer to provide useful energy at the site of substrate metabolism. [37]. A key component is an eternally vigilant sensor - a constitutively active kinase [50], which targets the near equilibrium reaction undertaken by (chlorideregulated) nucleoside diphosphate kinase (NDPK), a reaction that can occur at $1,000 / \mathrm{s}$ (see references in [35-37]). NDPK [10] has many isoforms forming a family of eight or nine human histidine kinases, which historically were thought to only perform nucleotide balancing roles with passing mention in biochemical textbooks [36]. This is no longer the case (http://www.dundee.ac.uk/mchs/ndpk). NDPK isoforms are found in many subcellular protein complexes with most in the cytosol and mitochondria [35], but some can even be secreted (mechanism unknown) or become nuclear where they can interact with hypersensitive sites on DNA and proteins mediating cell proliferation such as c-myc $[5,6]$. Some isoforms regulate microtubular assembly in a pathway important for the mode of action of anti-retroviral agents [11] and show complex regulation [4]. In Drosophila, NDPK protein is also known as AWD because its deletion causes abnormal wing discs, and in certain fly backgrounds, mutation of NDPK causes early lethality [39]. This conditional lethality is dependent on an interaction with an unusual class of cAMP-dependent phosphodiesterases that in turn interact with a hybrid zinc finger protein [39]. In the context of energy relays, human NDPK isoforms are found both in the matrix and the intermembrane space of mitochondria $[35,19]$. In yeast, NDPK is involved in a relay that maintains matrix GTP levels for 
mitochondrial iron uptake and TCA cycle function. NDPK in the inter-membrane space may facilitate efficient export of nucleotides from the mitochondrial matrix [1]. In the cytosol, some NDPK isoforms supply GTP to GTPases to maintain protein function, such as the endocytosis regulator dynamin [27], whereas others such as NDPK-B bind G proteins associated with the $\beta 2$-adrenoreceptor in the failing heart $[29,52]$. Thus, the NDPK family performs many conserved energy-related roles between the animal and plant kingdoms [16]. Importantly, in all cases, the relay of phosphate driven by NDPK occurs at near equilibrium conditions through the generation of phosphohistidine on a conserved histidine (H118 in human NDPK-A) that lies at the bottom of a deep cleft that binds the $\beta$ and $\gamma$ phosphates of nucleotides. CK2 phosphorylates NDPK close to this site, as discussed elsewhere [4].

NDPK binds AMPK We find that the ' $\mathrm{A}$ ' isoform of NDPK (NDPK-A, alternatively known as human nm23-H1 or mouse nm23-M1; henceforth called NDPK-A) binds one of the two isoforms of AMPK [37]. This raises the prospect of an "AMP kinase" with both AMP-independent and ATP-independent modes of operation. Could the hybrid NDPK-A/AMPK $\alpha 1$ kinase be part of a Watt governor complex? This is not the entire story because our most recent data suggest that other kinases are involved [50], but their complex interactions are beyond the scope of this review [4]. For completeness, the more speculative roles of these proteins [7-9] are discussed in Appendix 1. Nevertheless, it is clear that the epithelial apical membrane contains multi-protein complexes that consist of ion channels and protein kinases that regulate each other. Why? When consideration is given to the fact that PKA, PKC, NDPK, AMPK and CK2 together with local controlling phosphatases are all bound to CFTR, perhaps it is important to think beyond the current view of the CFTR as an ion channel modulated by multiple sites of phosphorylation by different protein kinases. Perhaps, CFTR provides a surface for kinase-kinase or kinase-phosphatase interactions. It is the view of this author that CFTR could also be an ideal timer by recycling its own energy sensor integrating signals at the membrane and cytosol, perhaps exchanging and recruiting different partners depending on the energy needs of the cell and the local ionic composition of chloride and sodium. Such a model invokes a Watt governor role for a CFTR-containing complex, but our data remain controversial because they are difficult to reproduce completely [7-9]. The only verifiable facts are that one isoform of NDPK and one isoform of AMPK bind one another and that the histidine phosphorylation of NDPK rises three- to fivefold when this interaction takes place in vitro [37]. A key problem (Fig. 1) is that there are many possible combinations of destinations for the ATP fed to AMPK other than CFTR itself (Fig. 2). Hallows et al. [22] suggest that AMPK-dependent phosphorylation of CFTR switches off CFTR channel function, but the

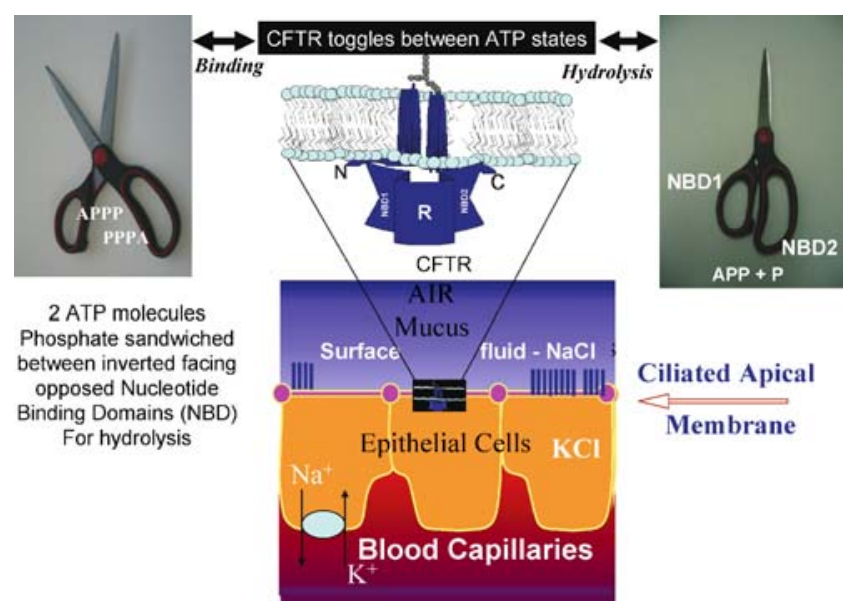

Fig. 1 CFTR modelled as a pair of scissors with each ATP trapped in a finger hole and sandwiched between the two ATP binding domains. Lipid encased CFTR is shown enlarged from the apical membrane bathed in surface fluid. In the exploded CFTR cartoon, the $R$ in the dark blue rectangle depicts the phosphorylated regulatory domain $(\mathrm{R}$ domain) whose exact location in three dimensions is unknown; the two scalloped rectangular structures behind the $\mathrm{R}$ domain are the nucleotide binding domains (NBD1 and 2, left and right) additionally depicted as finger handles in the scissor models (left and right) such that the central hinge in the scissors represents the $\mathrm{R}$ domain. Thus, the $\mathrm{R}$ domain only permits CFTR opening and closing provided the hinge is oiled (phosphorylation) and ATP is present between the NBDs. ATP trapping (slowed hydrolysis NBD1, on the left) and faster hydrolysis (perhaps NBD2, on the right) provides motive power to CFTR scissor blades to move dehydrated chloride by cutting a transient chloride/anion filled hole through the lipid bilayer (light blue) whilst generating a spring like action on the proximity of the ATP binding domains (shown as dark blue structure below transmembrane helices). The mechanism of force transmission to the transmembrane helices/scissor blades is unknown

exact residues and mechanism remain to be proven. An additional problem is that these authors [22] use cell permeant pharmacological activators of AMPK that either mimic AMP in structure, act by raising [AMP] indirectly by acting on upstream kinases or by poisoning mitochondrial function. Although CFTR may be phosphorylated by this manoeuvre (which has not been proven), the final target for AMPK on CFTR might differ depending on the route of activation, thus complicating this interpretation. Such a target might be a partner for AMPK such as NDPK [4, 37], which, at least, has the merit of showing ion-dependent histidine phosphorylation, albeit in vitro [36]. This is not an unreasonable idea if CFTR is really a sensor that must act differently in different energetic contexts. CFTR acts as a recruiter of so many proteins to the membrane that the CFTR vicinity must be crowded by multiple competing regulators, unless they interact after different stresses or in different subcelllular locations.

Is cystic fibrosis one or many diseases masquerading as a single entity? One of the problems with CF disease is that many paths can lead to a superficially identical phenotype. For example, the G551D mutation is an example that 


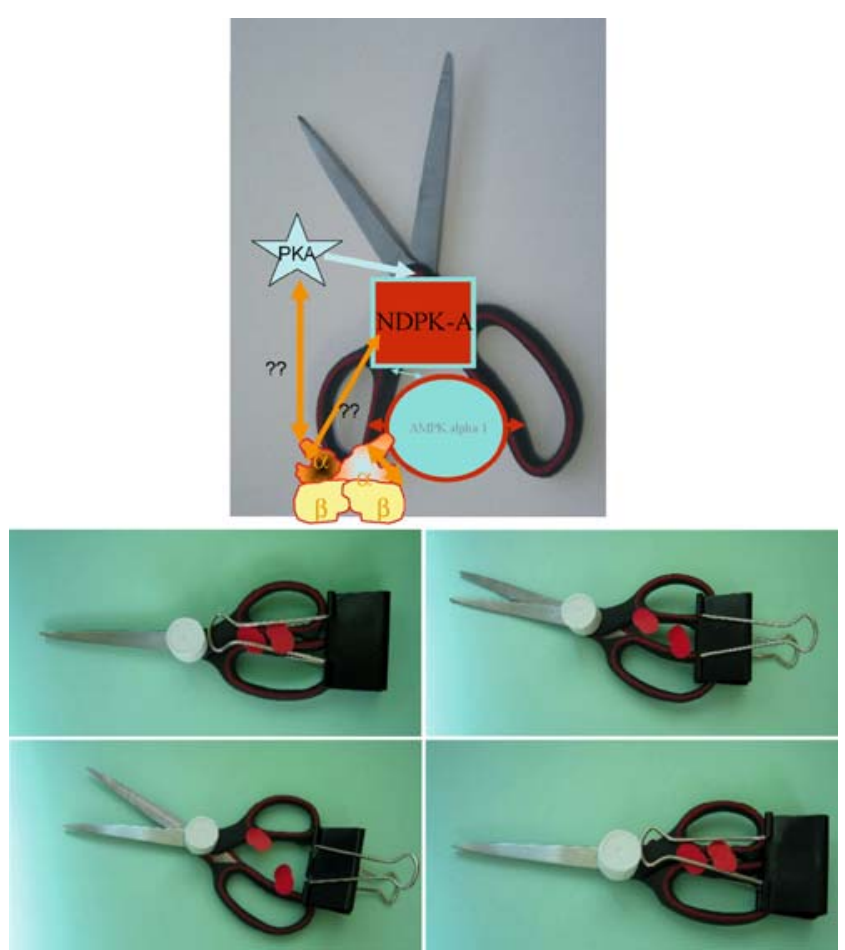

Fig. 2 Working model. Upper panel shows an overview with possible links between CK2, NDPK and AMPK using the scissor model of CFTR as shown in Fig. 1. Further analogies are shown in the lower four panels. In the lower analogy, The NDPK-A (white) is bound to the $\mathrm{R}$ domain hinge region of the scissors (CFTR representation as in earlier figures), whereas the paper clip (black) represents the heterotrimeric AMPK $\alpha 1$ (purely symbolically bound to NBDs, finger holes). The position of the silver steel handles on the clip changes in the panels representing possible interactions with ATP, NDPK-A and or CFTR. For clarity, CK2 is omitted from the lower panels, but note that NDPK is a CK2 target. The two red markers are ATP molecules in the sandwich of NBDs whose precise roles are not clear but their presence is required to invoke channel gating. The model is illustrative only. The sites of binding NDPK and AMPK are not necessarily those shown in the model whose purpose is to show possible interactions between kinases on a CFTR protein surface

creates a CFTR, which reaches its correct apical destination but then fails. What does failure mean? Imagine each ATP binding domain to be the finger hole in a pair of scissors (Fig. 1). In CFTR, the two nucleotide binding domains are likely to face one another, but in a head to tail manner, acting like opposed finger holes in a pair of scissors with ATP energy (rather than finger power) providing a molecular spring like action driving each finger hole towards the other and then springing apart. It must be the case that some molecular equivalent of the structure of the scissors and the (R domain) 'hinge' exist, transmitting the ATP-hydrolysed energy to the rest of CFTR or its associated partners. In G551D, for example, the G551 residue lies within a molecular lid that traps one gamma phosphate of ATP between the first and second nucleotide binding domains. The extra negative charge in G551D means that this lid is malformed, perhaps being more repulsive to phosphate. One can speculate that the timer that links ATP hydrolysis to CFTR function could go awry due to cap dysfunction. Thus, the phenotype may be the same, but the mechanism (and hence treatment) may be vastly different compared to $\triangle$ F508-CFTR as described below and in our recent paper [50].

The purpose of this review has been to invoke the new idea that interaction between protein kinases and CFTR may alter kinase function as opposed to the conventional view that describes actions of kinases on CFTR in its role as a channel. The former idea resonates with CFTR malfunction acting outside a purely channelopathy paradigm because one target kinase (NDPK) manifests common functions in development where fly genetics and mammalian epithelial biology overlap. For example, at first sight, references [1, 12, 14, 34, 39, 42] appear to be unrelated to one another. Closer scrutiny shows that NDPK dysfunction and endocytotic dynamin dysfunction produce related fly mutants in the AWD complex that malforms developing fly wings - the same AWD phenotype but different underlying molecular protein dysfunctions. Rikhy et al. [42] then link dynamin to cellular energetics and mitochrondrial function, which are all NDPK-regulated functions (albeit with different NDPK isoforms). Could these also be dysfunctional in cystic fibrosis (compare the work of Shapiro [46], Moudiou et al. [34] and Strandvik [48] with Rikhy et al. [42] and [6, 19])? Furthermore, NDPK dysfunction creates a coalescence of defects linking recycling of endosomes [27] to embryological defects [12] and energy dysfunction $[34,35,42]$. Crucially, we recently provided the first direct evidence for a protein-protein interaction that is not only selectively disrupted by the loss of F508 in CFTR but also creates a paradigm that predicts NDPK dysfunction [50]. In that study, we find that F508 and its surrounding amino acids act as part of a motif required to tether the catalytic subunit of a known NDPK-directed kinase, CK2 [4]. CK2 is a salt-regulated, heterodimeric kinase (CK2, formerly casein kinase 2), which creates a salt sensitive phenotype when knocked out in yeast. Importantly for the new model, CK2 is a key, recognised in vivo regulator of NDPK [50]. Even if nothing was known about CF physiology that pertained to ion transport, extrapolation of our findings would predict that salt sensing, CK2 dysfunction and NDPK dysfunction would be likely to be found in this disease.

Thus, most importantly, current ideas about the path from mutant CFTR to clinical disease invoke regulation by recruited kinases without asking whether CFTR regulates these recruits reciprocally. The author believes that both are required functions as would be demanded by a Watt governor to balance supply and demand of cellular energy (a Watt governor without feedback is useless). CK2 is a constitutively active kinase that could provide the necessary basal turnover of the governor when the epithelial 'engine' is idling and yet swiftly change its role via its partner 
kinases when the cellular engine needs full power. This idea resonates with process engineering principles: first, get your energy right (the art of the possible); then, get the entropy sorted (the art of the likely) and finally, engineer the most efficient solution (in biology, the Darwinian legacy). To put it in another way, using a post-diction (rather than a prediction) approach, if CFTR mutation did not dysregulate so many processes as it does, then CFTR would be a quite useless Watt governor would it not? Could this notion explain the complexity of cystic fibrosis?

Acknowledgements The author's laboratory is supported by the Wellcome Trust and Cystic Fibrosis Trust, and he is indebted to Dr Kate Treharne for discussions on many of the concepts explored in this paper. The review would never have arisen without my tutors in physics Tony Straddling and Jack Paton (Christ Church, Oxford) whose patient guidance in thermodynamics might one day benefit those suffering from cystic fibrosis.

\section{Appendix 1}

Wider roles of the NDPK protein (see http://www.dundee. ac.uk/mchs/ndpk).

In parallel, others $[2,3]$ working in energy- and fatrelated fields discovered an unexplained role for AMPK in the biogenesis of the power generating mitochondria [41]. This finding is discussed as part of a series of reviews on AMPK function recently published in the Journal of Physiology in 2006 (for example, [23, 41]) and will not be discussed further in this paper. From a multi-kinase perspective, this synthetic finding is relevant because NDPK has a long history of involvement in cellular proliferative events [4]. For example, NDPK plays an important role in signalling pathways downstream of growth receptors [6], and NDPK-A is a suppressor of metastasis (breast, cervix and melanoma). In mouse models, NDPK-A deletion induces excess neonatal mortality, low birth weight, poor growth and abnormal breast development. These functions are reviewed in a special issue of the Journal of Bioenergetics and Biomembranes and will not be described further in this paper [37]. Instead, the current review concentrates on potential 'signals' that integrate cell energy with ion concentration thereby updating a related review in Pflugers Archiv in 2001 on the means by which an epithelial cell membrane changes its histidine phosphorylation profile in response to ion species [49]. The new data reveals differential roles for CFTR [30], NDPK-A [37] and CK2 [4, 50] in mammalian histidine phosphorylation in the apical membrane [36] but remains poorly characterised primarily because of the lack of suitable tools for easy analysis outside specialised laboratories [37].

\section{References}

1. Amutha B, Pain D (2003) Nucleoside diphosphate kinase of Saccharomyces cerevisiae, Ynk1p: localization to the mitochondrial intermembrane space. Biochem J 370:805-815

2. Bandyopadhyay GK, Yu JG, Ofrecio J, Olefsky JM (2006) Increased Malonyl-CoA levels in muscle from obese and Type 2 diabetic subjects lead to decreased fatty acid oxidation and increased lipogenesis; thiazolidinedione treatment reverses these defects. Diabetes 55(8):2277-2285

3. Barnes K, Ingram JC, Porras OH, Barros LF, Hudson ER, Fryer LG, Foufelle F, Carling D, Hardie DG, Baldwin SA (2002) Activation of GLUT1 by metabolic and osmotic stress: potential involvement of AMP-activated protein kinase (AMPK). J Cell Sci 115:2433-2442

4. Biondi RM, Engel M, Sauane M, Welter C, Issinger OG, Jimenez de Asua L, Passeron S (1996) Inhibition of nucleoside diphosphate kinase activity by in vitro phosphorylation by protein kinase CK2. Differential phosphorylation of NDP kinases in HeLa cells in culture. FEBS Lett 399(1-2):183-187

5. Bosnar MH, De Gunzburg J, Bago R, Brecevic L, Weber I, Pavelic J (2004) Subcellular localization of A and B Nm23/NDPK subunits. Exp Cell Res 298(1):275-284

6. Braun S, Mauch C, Boukamp P, Werner S (2007) Novel roles of NM23 proteins in skin homeostasis, repair and disease. Oncogene 26(4):532-542

7. Crawford RM, Treharne KJ, Best OG, Muimo R, Reimen CE, Mehta A (2005) A novel physical and functional association between nucleoside diphosphate kinase $\mathrm{H} 1$ and AMP-activated protein kinase $\alpha 1$ in liver and lung. Biochem J 392(1):201-209

8. Crawford RM, Treharne KJ, Arnaud-Dabernat S, Daniel J-Y, Foretz M, Viollet B, Mehta A (2006) Understanding the molecular basis of the interaction between NDPK-A and AMPK $\alpha 1$. Mol Cell Biol 26(15):5921-5931

9. Crawford RM, Treharne KJ, Best OG, Riemen CE, Muimo R, Gruenert DC, Arnaud-Dabernat S, Daniel J-Y, Mehta A (2006b) NDPK-A (but not NDPK- B) and AMPK alpha 1 (but not alpha 2) binding CFTR in epithelial cell membranes. Cell Signal 18 (10):1595-603

10. Dabernat S, Larou M, Masse K, Dobremez E, Landry M, Mathieu C, Daniel J-Y (1999) Organization and expression of mouse nm23-MI gene. Comparison with $n m 23-M 2$ expression. Gene 236(2):221-230

11. D'Andrea G, Lizzi AR, Venditti S, Di Francesco L, Giorgi A, Mignogna G, Oratore A, Bozzi A (2006) Proteins pattern alteration in AZT-treated K562 cells detected by two-dimensional gel electrophoresis and peptide mass fingerprinting. Proteome Sci 4:4

12. Deitcher D (2001) Shibire's enhancer is cancer's suppressor. Trends Neurosci 11:625-626

13. Dinudom A, Harvey KF, Komwatana P, Jolliffe CN, Young JA, Kumar S, Cook DI (2001) Roles of the C termini of alpha -, beta -, and gamma -subunits of epithelial $\mathrm{Na}+$ channels $(\mathrm{ENaC})$ in regulating $\mathrm{ENaC}$ and mediating its inhibition by cytosolic $\mathrm{Na}^{+}$. J Biol Chem 276(17):13744-13749

14. Dinudom A, Fotia AB, Lefkowitz RJ, Young JA, Kumar S Cook DI (2004) The kinase Grk2 regulates Nedd4/Nedd4-2-dependent control of epithelial Na+ channels. Proc Natl Acad Sci U S A 101 (32):11886-11890

15. Dowd BF, Forbush B (2003) PASK (proline-alanine-rich STE20related kinase), a regulatory kinase of the $\mathrm{Na}-\mathrm{K}-\mathrm{Cl}$ cotransporter (NKCC1). J Biol Chem 278(30):27347-27353

16. Dorion S, Matton DP, Rivoal J (2006) Characterization of a cytosolic nucleoside diphosphate kinase associated with cell division and growth in potato. Planta 224(1):108-124

17. Dzeja PP, Terzic A (2003) Phosphotransfer networks and cellular energetics. J Exp Biol 206:2039-2047 
18. Dzeja PP, Terzic A, Wieringa B (2004) Phosphotransfer dynamics in skeletal muscle from creatine kinase gene-deleted mice. Mol Cell Biochem 256-257(1-2):13-27

19. Gordon DM, Lyver ER, Lesuisse E, Dancis A, Pain D (2006) GTP in the mitochondrial matrix plays a crucial role in organellar iron homeostasis. Biochem J 400(1):163-168

20. Gimenez I, Forbush B (2005) Regulatory phosphorylation sites in the NH2 terminus of the renal Na-K-Cl cotransporter (NKCC2). Am J Physiol Renal Physiol 289(6):F1341-F1345

21. Guggino WB, Stanton BA (2006) New insights into cystic fibrosis: molecular switches that regulate CFTR. Nat Rev Mol Cell Biol 7(6):426-436

22. Hallows KR, Kobinger GP, Wilson JM, Witters LA, Foskett JK (2003) Physiological modulation of CFTR activity by AMPactivated protein kinase in polarized T84 cells. Am J Physiol Cell Physiol 284(5):C1297-C1308

23. Hardie DG, Hawley SA, Scott JW (2006) AMP-activated protein kinase-development of the energy sensor concept. J Physiol 574 (Pt 1):7-15

24. Janssen E, Terzic A, Wieringa B, Dzeja PP (2003) Impaired intracellular energetic communication in muscles from creatine kinase and adenylate kinase (M-CK/AK1) double knock-out mice. J Biol Chem 278(33):30441-30449

25. Kato M, Warshel A (2005) Through the channel and around the channel: validating and comparing microscopic approaches for the evaluation of free energy profiles for ion penetration through ion channels. J Phys Chem B 109(41):19516-19522

26. Komwatana P, Dinudom A, Young JA, Co, o k DI (1998) Activators of epithelial $\mathrm{Na}+$ channels inhibit cytosolic feedback control. Evidence for the existence of a $\mathrm{G}$ protein-coupled receptor for cytosolic Na+. J Memb Biol 162(3):225-232

27. Krishnan KS, Rikhy R, Rao S, Shivalkar M, Mosko M, Narayanan R, Etter P, Estes PS, Ramaswami M (2001) Nucleoside diphosphate kinase, a source of GTP, is required for dynamindependent synaptic vesicle recycling. Neuron 30(1):197-210

28. Long YC, Zierath JR (2006) AMP-activated protein kinase signaling in metabolic regulation. J Clin Invest 116(7):1776-1783

29. Lutz S, Mura RA, Hippe HJ, Tiefenbacher C, Niroomand F (2003) Plasma membrane-associated nucleoside diphosphate kinase $(\mathrm{nm} 23)$ in the heart is regulated by beta-adrenergic signalling. Br J P harmacol 140(6):1019-1026

30. Mehta A (2005) CFTR: more than just a chloride channel. Pediatr Pulmonol 39:292-298

31. Mehta A, Bush A (2005) Beyond chloride transport: CFTR in the 21st century-introductory remarks to a new state of the art series. Pediatr Pulmonol 39:289-291

32. Milla CE, Billings J, Moran A (2005) Diabetes is associated with dramatically decreased survival in female but not male subjects with cystic fibrosis. Diabetes Care 28(9):2141-2144

33. Moran A, Basu R, Milla C, Jensen MD (2004) Insulin regulation of free fatty acid kinetics in adult cystic fibrosis patients with impaired glucose tolerance. Metabolism 53(11):1467-1472

34. Moudiou T, Galli-Tsinopoulou A, Vamvakoudis E, NousiaArvanitakis S (2007) Resting energy expenditure in cystic fibrosis as an indicator of disease severity. J Cyst Fibros 6(2):131-136

35. Muhonen WW, Lambeth DO (1995) The compartmentation of nucleoside diphosphate kinase in mitochondria. Comp Biochem Physiol B Biochem Mol Biol 110(1):211-223

36. Muimo R, Banner SJ, Marshall LJ, Mehta A (1998) Nucleoside diphosphate kinase and $\mathrm{Cl}(-)$-sensitive protein phosphorylation in apical membranes from ovine airway epithelium. Am J Respir Cell Mol Biol 18(2):270-278
37. Muimo R, Crawford RM, Mehta A (2006) NDPK-H1 in membranes of airway epithelia. J Bioenerg Biomembr 38(3-4): $181-187$

38. Puntheeranurak S, Schreiber R, Spitzner M, Ousingsawa J, Krishnamra N, Kunzelmann K (2007) Control of ion transport in mouse proximal and distal colon by prolactin. Cell Physiol Biochem 19(1-4):77-88

39. Provost E, Hersperger G, Timmons L, Ho WQ, Hersperger E, Alcazar R, Shearn A (2006) Loss-of-function mutations in a glutathione S-transferase suppress the prune-Killer of prune lethal interaction. Genetics 172(1):207-219

40. Rauh R, Dinudom A, Fotia AB, Paulides M, Kumar S, Korbmacher C, Cook DI (2006) Stimulation of the epithelial sodium channel $(\mathrm{ENaC})$ by the serum- and glucocorticoid-inducible kinase (Sgk) involves the PY motifs of the channel but is independent of sodium feedback inhibition. Pflugers Arch 452(3):290-299

41. Reznick RM, Shulman GI (2006) The role of AMP-activated protein kinase in mitochondrial biogenesis. J Physiol 574(Pt 1): 33-39

42. Rikhy R, Ramaswami M, Krishnan KS (2003) A temperaturesensitive allele of drosophila ses. $\beta$. reveals acute functions for the mitochrondrial adenine nucleotide translocase in synaptic transmission and dynamin regulation. Genetics 165(3):1243-1253

43. Schwiebert EM, Gesek F, Ercolani L, Wjasow C, Gruenert DC, Karlson K, Stanton BA (1994) Heterotrimeric G proteins, vesicle trafficking, and CFTR $\mathrm{Cl}^{-}$channels. Am J Physiol 267(1 Pt1): C272-C281

44. Selivanov VA, Alekseev AE, Hodgson DM, Dzeja PP, Terzic A (2004) Nucleotide-gated KATP channels integrated with creatine and adenylate kinases: amplification, tuning and sensing of energetic signals in the compartmentalized cellular environment. Mol Cell Biochem 256-257(1-2):243-256

45. Seong IS, Ivanova E, Lee JM, Choo YS, Fossale E, Anderson M, Gusella JF, Laramie JM, Myers RH, Lesort M, MacDonald ME (2005) HD CAG repeat implicates a dominant property of huntingtin in mitochondrial energy metabolism. Hum Mol Genet 14(19):2871-2880

46. Shapiro BL (1988) Mitochondrial dysfunction, energy expenditure and cystic fibrosis. Lancet 2(8605):289

47. Sims EJ, Green MW, Mehta A (2005) Decreased lung function in female but not male subjects with established cystic fibrosisrelated diabetes. Diabetes Care 28(7):1581-1587

48. Strandvik B (2004) Fatty acid metabolism in cystic fibrosis. N Engl J Med 350(6):605-607

49. Treharne KJ, Crawford RM, Mehta A (2006) Physiology of anion transport symposium paper: CFTR, chloride concentration and cell volume - could mammalian protein histidine phosphorylation play a latent role? Exp Physiol 91(1):131-139

50. Treharne KJ, Crawford RM, Xu Z, Chen JH, Best OG, Schulte EA, Gruenert DC, Wilson SM, Sheppard DN, Kunzelmann K, Mehta A (2007) Protein Kinase CK2, cystic fibrosis transmembrane conductance regulator and the (Delta)F508 Mutation: F508 deletion disrupts a kinase-binding site. J Biol Chem 282 (14):10804-10813

51. Villullas HM, Gonzalez ER (2005) A general treatment for the conductivity of electrolytes in the whole concentration range in aqueous and nonaqueous solutions. J Phys Chem B 109 (18):9166-9173

52. Wieland $T$ (2007) Interaction of nucleoside diphosphate kinase $B$ with heterotrimeric $\mathrm{G}$ protein betagamma dimers: consequences on $\mathrm{G}$ protein activation and stability. Naunyn Schmiedebergs Arch Pharmacol 374(5-6):373-383 